\title{
Allocution de clôture
}

\author{
J. Auber1 1
}

J'ai organisé le premier Symposium sur les Plé. coptères en 1956 à Lausanne. Il était seulement européen et nous n'étions que sept : H.B.N. Hynes (Liverpool), J. Illies (Schlitz), C. Consiglio (Rome), J. Raušer (Brno), P. Brinck (Lund), E. Pomeisl (Vienne) et moi-même. Comme le musée zoologique de Lau. sanne était en réparation et vu le nombre restreint de participants, j'avais organisé les séances dans notre villa de Lutry. Nous prenions les repas dans les restaurants de cette petite ville.

Aujourd'hui, 27 ans après, nous sommes un peu plus de 40 a participer au VIII symposium qui est vraiment une réunion mondiale : tous les continents sont représentés. Je suis heureux de rencontrer des émules en Espagne et en Italie, pays que j'ai eu par. ticulièrement de plaisir à visiter lorsque j'y récol. tais des Plécoptères.

De la morphologie et de la systématique, l'étude des Plécoptères a gagné d'autres domaines.

La zoogéographie et l'écologie occupent toujours une place prépondérante dans les exposés. On s'est penché, grâce au microscope électronique à balayage, sur les ultrastructures de diverses parties du corps et des œufs mettant en évidence leur intérêt phylogénétique. Quelques contributions ont aussi porté sur le développement des ceufs ou sur le tambourinage des mâles. Toutefois, je regrette de ne pas avoir entendu de nouvelles contributions au sujet de la morphologie des larvules ou de l'écologie de celles-ci. Mais, il s'agit là de domaines particulièrement difficiles.
Nous sommes ici dans la ville d'un zoologiste français qui a particulièrement contribué à la connaissance des Plécoptères dans la première moitié de ce siècle, le Professeur Raymond Despax (1886-1950). Vous me permettrez d'évoquer ici sa mémoire. Né et mort à Toulouse, Despax a fait ses études et sa carrière dans cette ville, sauf quelques brefs séjours dans d'autres villes, en particulier un stage de trois ans au Muséum d'Histoire Naturelle de Paris. Nommé d'abord préparateur à l'Université de Toulouse, il n'a pas tardé à y devenir professeur, chargé d'enseigner l'entomologie aux côtés de Vandel, le grand spécialiste de la parthénogénèse.

On doit à Despax un peu plus de cent publications dans des domaines variés de la zoologie : reptiles, batraciens, poissons et, parmi les insectes, sur les Trichoptères et les Ephéméroptères. Mais ce sont ses travaux sur les Plécoptères qui constituent la partie la plus intéressante de son œuvre, avec 35 publications.

Il a étudié surtout la faune des Pyrénées où il dis. posait d'un pied-à-terre avec le laboratoire d'hydrobiologie du lac d'Orédon dont il était le directeur. On lui doit de cette région toute une série d'especes nouvelles pour la science. Ses descriptions sont d'une grande rigueur et ses dessins d'une excellente qualité.

La partie la plus originale de son ceuvre est sa monographie des Isoperla qu'il appelait encore Chloroperla comme les auteurs du début du siècle. Il est le premier à montrer l'intérêt de l'armature sclérifièe du sac pénial du mâle pour préciser la nature des espèces dans ce genre difficile. Il est aussi le premier à proposer l'emploi de la notion de groupe d'especes dans la classification des Plécoptères.

J'ai été reçu par le professeur Despax en 1948 dans son laboratoire et à son domicile et j'ai gardé un sou-

1. Musée zoologique, $\mathrm{CH}$ - 1005 Lausanne, Suisse. 
venir ému de notre rencontre. J'étais fort flatté, tout jeune entomologiste, d'être reçu avec autant de cordialité par le maître d'alors de la Plécoptérologie. J'ai toujours vivement regretté de ne pas avoir pu le rencontrer plus tôt. En effet, j'avais préparé ma thèse pendant les années de la seconde guerre mondiale et, en Suisse, nous étions totalement isolés. I! ne nous était pas possible de correspondre avec les citoyens des pays en guerre. Je connaissais depuis le début de mes recherches les travaux de Despax qui était un peu devenu mon maître à penser. Hélas, notre première rencontre fut la seule. Despax décédait peu après des suites d'un accident vasculaire ${ }^{2}$.

2. Pour la liste des publications de R. Despax, voir Astre (G.). 1950. - Notice sur le Professeur Raymond Despax. Bull. Soc. Hist. nat. Toulouse, $85: 67-76+1$ pl. Pour les seules publications consacrées aux Plécoptères, Illies (J.). 1966. - Katalog der rezenten Ple. coptera. Das Tierreich, $82: \mathrm{xxx}+632 \mathrm{p}$. Walter de Gruyter \& $\mathrm{Co}$. Berlin. 